\section{Associação da Cor da Pele com Diabetes Mellitus Tipo 2 e Intolerância à Glicose em Mulberes Obesas de Salvador, Babia}

\section{RESUMO}

Fora do Brasil a prevalência de diabetes mellitus (DM) tipo 2 é maior em negros do que em brancos. Mulheres do ambulatório de obesidade do Hospital Universitário Professor Edgard Santos, Salvador - BA, foram estudadas para avaliar se a cor de pele escura tem prevalência mais alta de DM tipo 2 e de intolerância à glicose (ITG), com diagnóstico baseado nos critérios da Organização Mundial da Saúde, independentemente da idade e do índice de massa corporal (IMC). A cor da pele foi classificada como clara $(n=166)$, intermediária $(n=186)$ ou escura $(n=128)$. Determinouse o nível de glicose plasmática pelo método da glicose-oxidase. O teste de tolerância à glicose oral foi realizado em todas as pacientes, com exceção de 14 que já sabiam ser diabéticas. Pacientes com pele clara, intermediária e escura foram semelhantes em idade, IMC e relação cintura/quadril. A prevalência de DM, contudo, foi significantemente maior $(\mathrm{p}<0,05)$ nas de pele escura $(13,3 \%)$ quando comparadas com as de pele clara (7,2\%). A prevalência de ITG foi também maior, embora estatisticamente não significante $(\mathrm{p}=0,088)$, nas de pele escura $(18,8 \%)$ do que nas de pele clara (12,7\%). A relação entre o número de pacientes com e sem o diagnóstico de DM ou ITG foi 1,9 vezes maior no grupo pele escura, comparado com clara (odds ratio $(O R)=1,9$; intervalo de confiança (IC) $95 \%=$ 1, 1-3,2; $p<0,05$ ). Esta associação não foi alterada (OR= 1,7; IC 95\%=1,0-2,9) e permaneceu moderadamente significante $(p=0,069)$, mesmo após 0 ajuste para idade e IMC, através de regressão logística. Neste estudo, as mulheres obesas com pele escura tiveram maior associação com DM e ITG, independentemente da idade e IMC. (Arq Bras Éndocrinol Metab $2001 ; 45 / 5: 475-480$ )

Unitermos: Diabetes tipo 2; Intolerância a glicose; Obesidade; Cor de pele; Raça.

\begin{abstract}
Outside Brazil the prevalence of type 2 diabetes mellitus (DM) is higher in blacks than in whites. Women from the obesity outpatient clinic of Professor Edgard Santos University Hospital, Salvador - BA, were studied, to assess whether dark skin color have higher prevalence of type $2 \mathrm{DM}$ and impaired glucose tolerance (IGT), diagnosis based on the World Health Organization criteria, independently of age and body mass index (BMI). Skin color was classified as light $(n=166)$, medium $(n=186)$ and dark $(n=128)$. Plasma glucose level was determined by the glucose oxidase method. Oral glucose tolerance test was performed in all patients, except for 14 that were known to have DM. Patients with light, medium and dark skin color were similar regarding age, BMl and waist/hip ratio. The prevalence of DM, however, was significantly higher $(\mathrm{p}<0.05)$ in women with dark (13.3\%) as compared with light skin (7.2\%). The prevalence of IGT was also higher, although statistically not significant $(p=0.088)$, in patients with dark (18.8\%) than with light $(12.7 \%)$ skin. The odds of receiving the diagnosis DM or IGT was 1.9 times higher in women with dark than with light skin (odds ratio $(O R)=1.9,95 \%$ confidence interval $(\mathrm{Cl})=1.1-3.2, \mathrm{p}<0.05)$. This association was not importantly altered $(\mathrm{OR}=1.7$, $95 \% \mathrm{Cl}=1.0-2.9)$ and remained marginally significant $(\mathrm{p}=0.069)$, even after the logistic regression adjustment for age and BMl. In this study, obese
\end{abstract}

\section{artigo original}

\author{
Itana Coutinho Brito \\ Antônio Alberto Lopes \\ Leila Maria Batista Araíjo
}

Hospital Professor Edgard Santos, Universidade Federal da Bahia (UFBA), Salvador, $B A$. 
women with dark skin color had higher association with DM and IGT, independently of age and BMI. (Arq Bras Endocrinol Metab 2001;45/5:475-480)

Keywords: Type 2 diabetes; Impaired glucose tolerance; Obesity; Skin color; Race.

$\mathrm{N}$ O BRASIL, EXISTE UMA GRANDE mistura racial devido ao cruzamento dos índios nativos, dos colonizadores brancos europeus e dos negros africanos, particularmente do oeste da África. Em Salvador, capital da Bahia, predomina a mistura do branco com o negro, sendo a população composta, segundo os dados do Instituto Brasileiro de Geografia e Estatística (IBGE), de $17 \%$ de brancos, $22 \%$ de negros e $60 \%$ de mulatos (indivíduos com traços fenotípicos derivados da miscigenação entre negros e brancos) (1). Na Bahia existe evidência de que a mistura racial envolvendo o negro africano é importante, mesmo entre indivíduos considerados brancos (2). A predisposição para distúrbios metabólicos nesta população seria herdada do oeste africano, especialmente dos bantus e sudameses. Outros fatores, como o grau e tipo de obesidade, a atividade física e o nível sócio-econômico também têm importância como fatores de risco para desenvolvimento de diabetes mellitus (DM).

Malerbi e cols. (3), num estudo multicêntrico no Brasil, mostraram que a prevalência de DM estimada foi de $7,4 \%$ e a de intolerantes à glicose (ITG) de $7,7 \%$, sendo esta freqüência cerca de duas vezes maior em obesos. As prevalências de DM e ITG em relação à cor da pele foram de $7,5 \%$ e $7,8 \%$, respectivamente, nos indivíduos brancos ( $64 \%$ da amostra) e de $7,1 \%$ e 7,6\% em indivíduos de outros grupos raciais, denominados não brancos.

Diversos estudos realizados em outros países mostram que a prevalência de DM tipo 2 (DM2) e ITG é maior entre os negros do que entre os indivíduos da raça branca (4-12). Ainda não estão bem estabelecidas as razões destas diferenças raciais. Alguns estudos indicam que fatores ambientais e comportamentais têm um papel importante como determinante no desenvolvimento de DM2 $(4,5,9,13-15)$. Isto é exemplificado pela maior prevalência de DM2 nos japoneses que moram nos EUA do que naqueles que vivem em Hiroshima, no Japão (12). Esta prevalência de DM seria, portanto, determinada por interação de fatores genéticos, ambientais e culturais. Alguns autores defendem que o mecanismo primário para o desenvolvimento de DM2 nos negros seria a hiperinsulinemia e a resistência à insulina.
O objetivo deste estudo é avaliar se a cor da pele está associada com as prevalências de DM2 e ITG em uma amostra de mulheres da cidade de Salvador, Bahia, independentemente da idade e do índice de massa corpórea (IMC).

\section{CASUÍSTICA E MÉTODOS}

Foram estudadas 480 pacientes obesas que procuraram voluntariamente o ambulatório de obesidade, anexo ao Hospital Universitário Professor Edgard Santos (HUPES), em Salvador - BA, no período de 1993 a 2000, para perda de peso. Excluímos os pacientes do sexo masculino pelo reduzido número ( $<7 \%$ do total de pacientes). Não foram também selecionados os que tinham alguma doença crônica, exceto hipertensão ou dislipidemia relacionadas à síndrome pluri-metabólica; os que já estavam em dieta hipocalórica; e os que já faziam uso de medicações que podiam interferir no metabolismo glicídico. Quatorze pacientes (3\%) já tinham apresentado glicemias elevadas no passado.

A classificação de raça que tem sido utilizada no ambulatório de obesidade foi orientada por um dos autores (LMBA), seguindo os critérios de Krieger e cols., modificados por Azevêdo (16,17). Esta classificação leva em consideração basicamente traços fenotípicos externos: a cor da pele, a forma do nariz, espessura dos lábios e textura dos cabelos. Segundo esta classificação, as pacientes foram inicialmente agrupadas em cinco categorias: branco, mulato claro, mulato médio, mulato escuro e negro. Devido ao reduzido número de brancos e negros no ambulatório e a dificuldade em distinguir o mulato claro, ou moreno, do branco e mulato escuro do negro, optou-se em classificar os indivíduos considerando-se fundamentalmente a cor da pele: clara (branco e mulato claro), intermediária (mulato médio) e escura (mulato escuro e preto).

A medida da cintura foi obtida com a mensuração do menor diâmetro da cintura entre o rebordo costal e crista ilíaca e a dos quadris no seu maior diâmetro.

Todas as pacientes, exceto as que já tinham glicemia de jejum elevada, foram orientadas a manter dieta com mais de $150 \mathrm{~g}$ de carboidratos até serem submetidas ao teste de tolerância à glicose oral com $75 \mathrm{~g}$ de glicose, em jejum, e colhido o plasma para determinação de glicose nos tempos 0,1 e $2 \mathrm{~h}$ pelo método da glicose-oxidase. As pacientes hipertensas em uso de diurético foram orientadas a descontinuar a medicação três dias antes do teste.

Para o diagnóstico de DM2 e de ITG foram utilizados os critérios da Organização Mundial de Saúde (OMS) (18).

Arq Bras Endocrinol Metab vol $45 n^{\circ} 5$ Outubro 2001 
Utilizou-se análise de variância (ANOVA) para comparar a idade, índice de massa corporal (IMC) e relação cintura/quadril entre os grupos com pele clara, intermediária e escura. Para comparar a prevalência de DM e ITG entre os grupos foi utilizado o teste do quiquadrado. Análise de regressão logística foi utilizada para estimar odds ratios (OR) da associação entre cor da pele e evento (DM e ITG), ajustadas para idade $\left(<40\right.$ versus $\geq 40$ anos) e IMC ( $<40$ versus $\left.\geq 40 \mathrm{~kg} / \mathrm{m}^{2}\right)$. O software Statistical Package for Social Science (SPSS), versão 6.0 para Windows foi utilizado para análise estatística (20).

\section{RESULTADOS}

A cor da pele das 480 participantes foi classificada como clara em 34,6\%, intermediária em 38,8\% e escura em $26,7 \%$. A idade na amostra total apresentou variação entre 14 e 68 anos, média $( \pm \mathrm{DP})$ de $36 \pm 10$ anos e mediana de 36 anos. O IMC variou entre 30,3 e $71,9 \mathrm{~kg} / \mathrm{m}^{2}$, média $( \pm \mathrm{DP})$ de $40,4 \pm 6,4 \mathrm{~kg} / \mathrm{m}^{2}$ e mediana de $39 \mathrm{~kg} / \mathrm{m}^{2}$.

A tabela 1 mostra as características clínicas das pacientes de acordo com a cor da pele. Os grupos de cor de pele clara, intermediária e escura foram semelhantes quanto à idade, ao IMC e à relação cintura/quadril.

A tabela 2 mostra a distribuição dos pacientes de acordo com a cor da pele e a resposta ao teste de tolerância à glicose, segundo os critérios da OMS. O DM2 foi diagnosticado em $9,8 \%$ ou em 47 das 480 pacientes, correspondendo a 13,3\% (17/128) das mulheres de cor de pele escura, 9,7\% (18/186) de cor intermediária e 7,2\% (12/166) da cor clara. A comparação entre os grupos mostrou que a freqüência de
Tabela 2. Prevalência de diabetes mellitus (DM) ou intolerância à glicose (ITG) no grupo total e por estratos de cor de pele. Valores em percentual (\%) e número (N).

\begin{tabular}{lccccc}
\hline & \multicolumn{4}{c}{ Cor de Pele } \\
\cline { 2 - 5 } Diagnóstico & Clara & Intermediária & Escura & Total \\
\hline DM & $\%(N)$ & $7,2(12)$ & $9,7(18)$ & $13,3(17)^{*}$ & $9,8(47)$ \\
ITG & $\%(N)$ & $12,7(21)$ & $11,8(22)$ & $18,8(24)^{\star *}$ & $14,0(67)$ \\
Normal & $\%(N)$ & $80,1(133)$ & $78,5(146)$ & $68,0(87)$ & $76,3(366)$ \\
\hline
\end{tabular}

* $p=0,050$ comparando pele escura e clara

** $p=0,088$ comparando pele escura e clara

DM2 em mulheres de cor escura foi significativamente maior do que nas de pele clara $(\mathrm{p}=0,05)$. A presença de ITG foi diagnosticada em $13,9 \%(67 / 480)$ das pacientes, sendo mais freqüente nas mulheres de pele escura $(18,8 \%$ ou $24 / 128)$ do que nas de pele clara $(12,7 \%$ ou $21 / 166) ; p=0,088$.

A tabela 3 mostra a freqüência de pacientes com ambos os distúrbios metabólicos (DM2 ou ITG) de acordo com a cor da pele e as associações não ajustadas e ajustadas para idade e IMC. Foi observada uma associação estatisticamente significante ( $\mathrm{p}$ para tendência= 0,019 ) entre intensidade da cor da pele e aumento da prevalência de DM ou ITG. Esta prevalência foi significativamente maior $(\mathrm{p}<0,05)$ nas mulheres de pele escura $(32,0 \%)$ do que nas de pele clara $(19,9 \%)$.

A relação entre o número de pacientes com DM ou ITG e o número de pacientes livres do evento foi 1,9 vezes maior no grupo com pele escura do que no grupo de pele clara $(\mathrm{OR}=1,9$; IC $95 \%=1,1-3,2$; $\mathrm{p}<0,05)$. Esta associação não se alterou de forma importante $(\mathrm{OR}=1,7$; IC $95 \%=1,0-2,9)$ quando se ajustou

Tabela 1. Características clínicas das mulheres obesas em relação à cor da pele.

\begin{tabular}{|c|c|c|c|c|c|}
\hline \multirow[b]{3}{*}{ Característica } & \multicolumn{4}{|c|}{ Cor da Pele } & \multirow{3}{*}{ Valor de $\mathrm{p}^{*}$} \\
\hline & Clara & Intermediária & Escura & Total & \\
\hline & $N=166$ & $N=186$ & $N=128$ & $N=480$ & \\
\hline $\begin{array}{l}\text { ldade (anos) } \\
\text { Média } \pm D P \\
\text { Mediana }\end{array}$ & $\begin{array}{c}35,3 \pm 10,7 \\
35\end{array}$ & $\begin{array}{c}35,8 \pm 9,2 \\
36\end{array}$ & $\begin{array}{c}37,0 \pm 10,3 \\
37\end{array}$ & $\begin{array}{c}36,0 \pm 10,0 \\
36\end{array}$ & 0,312 \\
\hline $\begin{array}{l}\text { IMC }\left(\mathrm{kg} / \mathrm{m}^{2}\right) \\
\text { Média } \pm \mathrm{DP} \\
\text { Mediana }\end{array}$ & $\begin{array}{c}39,9 \pm 6,9 \\
38,4\end{array}$ & $\begin{array}{c}40,4 \pm 6,2 \\
39,0\end{array}$ & $\begin{array}{c}40,8 \pm 5,8 \\
40,2\end{array}$ & $\begin{array}{c}40,4 \pm 6,4 \\
39,0\end{array}$ & 0,507 \\
\hline $\begin{array}{l}\text { Cintura/Quadril } \\
\text { Média } \pm D P \\
\text { Mediana }\end{array}$ & $\begin{array}{c}0,84 \pm 0,06 \\
0,84\end{array}$ & $\begin{array}{c}0,83 \pm 0,07 \\
0,83\end{array}$ & $\begin{array}{c}0,84 \pm 0,06 \\
0,84\end{array}$ & $\begin{array}{c}0,84 \pm 0,06 \\
0,84\end{array}$ & 0,510 \\
\hline
\end{tabular}


Tabela 3. Associação entre cor da pele e prevalência de diabetes mellitus ou intolerância à glicose nas mulheres obesas não ajustada e ajustada para idade, IMC e ambas através de análise de regressão logística.

\begin{tabular}{|c|c|c|c|c|c|c|c|}
\hline \multirow[b]{2}{*}{ Cor de Pele } & \multicolumn{3}{|c|}{ DM ou ITG } & \multicolumn{4}{|c|}{ Odds ratio (IC+ 95\%) } \\
\hline & Sim & Não & $\%^{++}$ & Não ajustada & $\begin{array}{c}\text { Ajustada } \\
\text { para idade }\end{array}$ & $\begin{array}{l}\text { Ajustada } \\
\text { para IMC }\end{array}$ & $\begin{array}{l}\text { Ajustada para } \\
\text { IMC e idade }\end{array}$ \\
\hline $\begin{array}{l}\text { Clara } \\
\text { Intermediária } \\
\text { Escura }\end{array}$ & $\begin{array}{l}33 \\
40 \\
41\end{array}$ & $\begin{array}{c}133 \\
146 \\
87\end{array}$ & $\begin{array}{l}19,9 \\
21,5 \\
32,0\end{array}$ & $\begin{array}{c}\text { Referência } \\
1,1(0,7-1,8) \\
1,9(1,1-3,2)^{*}\end{array}$ & $\begin{array}{c}\text { Referência } \\
1,1(0,6-1,8) \\
1,9(1,1-3,2)^{*}\end{array}$ & $\begin{array}{c}\text { Referência } \\
1,1(0,6-1,7) \\
1,7(1,0-2,9)^{\star *}\end{array}$ & $\begin{array}{c}\text { Referência } \\
1,1(0,6-1,7) \\
1,7(1,0-2,9)^{\star *}\end{array}$ \\
\hline
\end{tabular}

+ $\mathrm{IC}=$ intervalo de confiança; ${ }^{++} \mathrm{p}$; para tendência= 0,019;

${ }^{*} p<0,05$ comparado com pele clara; ${ }^{* *} p<0,07$ comparado com pele clara.

para idade e se manteve moderadamente significante $(\mathrm{p}=0,069)$ após o ajuste simultâneo para idade e IMC.

\section{DISCUSSÃO}

Os dados obtidos neste estudo indicam que a freqüência de DM e ITG é maior entre as obesas de cor de pele escura, em relação às de pele intermediária e às de pele clara, com diferença estatisticamente significante, especialmente quando se somaram os dois tipos de distúrbios (tabela 3).

Malerbi e cols. (3) analisaram uma amostra de 21.847 indivíduos de ambos os sexos, com idades entre 30 e 69 anos, em 9 cidades brasileiras, de novembro/86 a julho/88 e observaram que as prevalências de DM e ITG foram similares entre bran$\cos (7,5$ e $7,1 \%$, respectivamente $)$ e não brancos $(7,8$ e $7,6 \%)$. Este estudo considerou apenas cor da pele branca ( $64 \%$ da população estudada) e não branca para classificação racial e incluiu DM tipo 1 e tipo 2. Além do mais, apenas os pacientes suspeitos de DM ou ITG foram submetidos ao teste de tolerância à glicose.

Diversos estudos mostram maior prevalência de DM em negros quando comparado com brancos (412). Harris e cols. (5) avaliaram uma amostra representativa da população dos EUA de 1.460 indivíduos, $52,2 \%$ mulheres e $47,8 \%$ homens, na faixa etária de 20 74 anos, incluindo $13,7 \%$ de obesos, e observaram que a prevalência de DM foi 7,3\% em mulheres brancas e $11,0 \%$ em mulheres negras, com diferença estatisticamente significante. Lipton e cols. (7) acompanharam 11.097 indivíduos brancos e negros, com idades entre 25 e 70 anos, durante 16 anos (1971-1987), incluindo $32 \%$ obesos, e observaram que a incidência de DM ajustada para idade foi $15 \%$ entre mulheres negras e $7 \%$ entre mulheres brancas, mostrando a tendência de maior incidência de DM nas negras. Cowie e cols. (8) avaliaram 4.379 indivíduos de ambos os sexos, com idades entre 20 a 74 anos, durante 5 anos (1976-1980) e observaram que a prevalência de DM foi $8,7 \%$ e 5,3\% respectivamente em homens negros e brancos e 10,4\% e $6,8 \%$ em mulheres negras e brancas respectivamente, cuja diferença foi significante $(\mathrm{p}<0,001)$. No estudo de Brancati e cols. (12), em 2.646 africanos e 9.461 brancos seguidos por nove anos, a incidência de DM foi maior em mulheres ( 2,4 vezes) e em homens ( 1,5 vezes) negros, respectivamente, do que nos brancos.

Nos indivíduos que já têm uma predisposição genética ao DM, fatores ambientais e comportamentais têm um papel importante na determinação desta doença. Isto é evidenciado em vários estudos: King \& Rewers (4) e Osei \& Schuster (9) mostraram maior prevalência de DM e ITG nos afro-americanos quando comparados aos brancos americanos ou nativos africanos; Fujimoto e cols. (13) relataram maior prevalência destes distúrbios do metabolismo glicídico nos japoneses americanos do que entre a população branca dos EUA ou japoneses que vivem em Hiroshima, Japão; O'Dea e cols. (14) descreveram uma prevalência 15 vezes maior de DM2 numa comunidade australiana aborígene com uma longa história de aculturação quando comparada à população australiana.

Estes estudos sugerem que com a mudança de ambiente (rural para urbana, áreas não industrializadas para áreas industrializadas), os indivíduos mudam seu estilo de vida: modificam sua dieta (maior ingestão de gordura), sua atividade física (vida mais sedentária) e, conseqüentemente, passam a ganhar peso, tornando-se mais resistentes à insulina e hiperinsulinêmicos $(4,6,9)$.

Entre os fatores de risco para o desenvolvimento de DM2, a obesidade é a que está mais fortemente relacionada. Cercato e cols. (20) estudaram 474 indivíduos obesos, $86,9 \%$ do sexo feminino e 13,1\% do sexo masculino, no Hospital das Clínicas da FMUSP, durante os anos de 1997 a 1999, e observaram que a prevalência de DM foi de $15,4 \%$, havendo uma correlação positiva entre o aumento do IMC e a ocorrência de DM. 
Não apenas o grau de obesidade, mas também o tipo de distribuição de gordura pode influenciar no aparecimento de diabetes num indivíduo geneticamente predisposto: a maior proporção de gordura central em relação à gordura periférica implica em maior resistência à insulina, hiperinsulinemia e intolerância à glicose. Segundo alguns autores (22-25), as mulheres negras apresentam maior índice cintura/quadril do que as brancas, possivelmente determinado geneticamente, $\mathrm{e}$ isto poderia influenciar na maior prevalência de distúrbios metabólicos. Entretanto, Dowling \& PiSunyer (21) observaram que a relação obesidade central, hiperinsulinemia, resistência à insulina, e intolerância à glicose ocorria com maior freqüência nas mulheres brancas quando comparada às negras. Albu \& Pi-Sunyer (22) notaram que estes distúrbios metabólicos estavam mais relacionados à gordura visceral, avaliada por tomografia computadorizada, que por sua vez era menor nas mulheres negras do que nas brancas. Resnick e cols. (10) relataram que o efeito do IMC no risco de DM é diferente entre as raças, havendo maior risco nos negros do que nos brancos com IMC baixo $\left(22 \mathrm{~kg} / \mathrm{m}^{2}\right)$ e um risco equivalente em ambos os grupos com IMC alto $\left(32 \mathrm{~kg} / \mathrm{m}^{2}\right)$, sugerindo um menor grau de gordura visceral entre os obesos negros ou um maior impacto da gordura visceral nos obesos brancos.

Alguns autores (23-27) sustentam a hipótese da resistência à insulina / exaustão pancreática: a resistência crônica à insulina levaria a uma hipersecreção de insulina, com exaustão das células beta e conseqüente hiperglicemia. Estudos em adultos mostram que os negros são mais hiperinsulinêmicos e resistentes à insulina do que os brancos, não havendo relação significante com o índice de massa corporal (IMC), com a distribuição de gordura ou fatores comportamentais, sendo este hiperinsulinismo atribuído a uma herança genética $(12,15)$. Osei e cols. (26) observaram que os negros descendentes de africanos têm maior concentração de insulina, iguais concentrações de peptídio C e extração hepática de insulina e clearance de insulina diminuídos, quando comparados aos brancos americanos; isto implica que o hiperinsulinismo nos negros não pode ser atribuído a uma hipersecreção das células beta, mas sim às alterações da extração hepática de insulina e do clearance de insulina, determinados talvez por fatores genéticos, uma vez que estas alterações foram independentes do IMC e distribuição corporal de gordura. Estudos em crianças e adolescentes reforçam esta hipótese. As crianças negras têm maior secreção de insulina em resposta a uma ingestão oral de glicose quando comparadas às brancas, em todos estágios da puberdade (27-29). As crianças com altos níveis de insulina são de alto risco para desenvolver DM tipo 2 mais tarde na vida e promover o desenvolvimento de complicações desta doença antes do diagnóstico.

Observou-se no presente estudo que $70 \%$ das obesas diabéticas desconheciam este diagnóstico à primeira consulta. Considerando a importância do diagnóstico precoce de DM e ITG, justifica-se a inclusão de glicemia de jejum e/ou teste de tolerância à glicose oral na investigação laboratorial do indivíduo obeso (30). No estudo de Mannucci e cols. (31), em 528 obesos ( $84 \%$ de mulheres) foi comparada a freqüência de DM e ITG pelos critérios da OMS e de DM e glicemia de jejum alterada pelo critério da Associação Americana de Diabetes, e concluíram que a glicemia de jejum maior que $126 \mathrm{mg} \%$ apenas não mostra sensibilidade para o screening de DM em obesos. Uma vez diagnosticada esta condição, tornam-se necessárias medidas terapêuticas e preventivas para diminuir o risco de doença cardiovascular e de complicações microvasculares relacionadas à hiperglicemia pós-prandial. Concluindo, observamos maior freqüência de distúrbios do metabolismo do hidrato de carbono em mulheres obesas de cor de pele escura na população estudada, cujas razões não são bem conhecidas. São necessários estudos epidemiológicos mais direcionados para tentar identificar as diferentes características clínicas, laboratoriais e ambientais que seriam responsáveis por estas alterações.

\section{AGRADECIMENTOS}

Ao $\mathrm{CNPq}$ pela bolsa de auxílio integrado recebida (Processo no. 523.837/95-0); a Aldenice Viana, técnica de laboratório, pela coleta dos testes de tolerância a glicose; a Ivanise Maria Santana Silva, secretária, pela dedicada assistência.

\section{REFERÊNCIAS}

1. Instituto Brasileiro de Geografia e Estatística. Pesquisa Nacional por Amostragem de Domicilios (PNAD): Bahia e Região Metropolitana de Salvador, 1998

2. Sousa MGF, Azevêdo ES, Silva MCBO, Freire NBVM. Brancos descendentes de negros na sociedade brasileira. Ciência e Cultura 1987;39:1186-8.

3. Malerbi DA, Franco LJ. Multicenter study of the prevalence of diabetes mellitus and impaired glucose tolerance in the urban Brazilian population aged 30-69 yr. Diabetes Care 1992; 15:1509-16.

4. King $\mathrm{H}$, Rewers M. Global estimates for prevalence of diabetes mellitus and impaired glucose tolerance in adults. Diabetes Care 1993;16:157-77. 
5. Harris Ml, Hadden WC, Knowler WC, Bennett PH. Prevalence of diabetes and impaired glucose tolerance and plasma glucose levels in U.S. population aged 20-74 yr. Diabetes 1987:36:23-34.

6. O'Brien TR, Flanders D, Decoufle P, Boyle CA, DeStefano $F$, Teutsch $S$. Are racial differences in the prevalence of diabetes in adults explained by differences in obesity? JAMA 1989:262:1485-8.

7. Lipton RB, Liao Y, Cao G, Cooper RS, McGee D. Determinants of incident non-insulin dependent diabetes mellitus among blacks and whites in a national sample: The NHANES I Epidemiologic Follow-up Study. Am J Epidemiol 1993;138:826-39.

8. Cowie CC, Harris MI, Silverman RE, Johnson EW, Rust KF. Effect of multiple risk factors on differences between blacks and whites in the prevalence of non-insulindependent diabetes mellitus in the United States. Am J Epidemiol 1993; 137:719-32.

9. Osei K, Schuster DP. Metabolic characteristics of African descendants: a comparative study of African-Americans and Guanaian immigrants using minimal model analysis. Diabetologia 1995;38:1 103-9.

10. Resnick HE, Valsania P, Halter JB, Lin X. Differential effects of BMl on diabetes risk among black and white Americans. Diabetes Care 1998;21:1828-35.

11. Winkleby MA, Kraemer HC, Akn DK, Varady NA. Ethnic and socioeconomic differences in cardiovascular disease risk factors. JAMA 1998;280:356-62.

12. Brancati FL, Linda Kao WH, Folsom AR, Warson RL, Szklo $\mathrm{M}$. Incident type 2 diabetes mellitus in African-American and white adults: the Atherosclerosis Risk in Communities Study. JAMA 2000;283:2253-9.

13. Fujimoto WY, Leonetti DL, Kinyoun JL, Newell-Morris L, Shuman WP, Stolov WC, et al. Prevalence of diabetes mellitus and impaired glucose tolerance among second generation Japanese-American men. Diabetes 1987;36: 721-9.

14. O’Dea K, Patel M, Kubisch D, Hopper J, Traianedes K. Obesity, diabetes, and hyperlipidemia in a central Australian aboriginal community with a long history of acculturation. Diabetes Care 1993;16:1004-10.

15. Pan XR, Yang WY, Li GW, Liu J. Prevalence of diabetes and its risks factors in China, 1994. Diabetes Care 1997:20:1664-9.

16. Krieger H, Morton NE, Azevêdo ES, Freire-Maia A, Yasuda N. Racial admixture in North Eastern Brazil. Ann Hum Genet 1965;29:113-25.

17. Azevêdo ES. Subgroup studies of black admixture within a mixed population of Bahia, Brazil. Ann Hum Genet 1980;44:55-60

18. World Health Organization. Diabetes Mellitus: Report of a WHO Study Group. Technical Report Series, 727, Geneva: WHO 1985

19. Norusis. MJ SPSS for Windows: Base system user's guide, release 6.0. Chicago, IL: SPSS Inc, 1993
20. Cercato C, Silva S, Sato A, Mancini M, Halpern A. Risco cardiovascular em uma população de obesos. Arq Bras Endocrinol Metab 2000;44(supl1):45-8.

21. Dowling HJ, Pi-Sunyer FX. Race-dependent health risks of upper body obesity. Diabetes 1993:42:537-43.

22. Albu JB, Murphy L, Frager DH, Johnson JA, Pi-Sunyer FX. Visceral fat and race-dependent health risks in obese non-diabetic premenopausal women. Diabetes 1997; 46:456-62.

23. Reaven GM. Role of insulin resistance in human disease. Diabetes 1998;37:1595-607.

24. Haffner SM, Stern MP, Mitchell BD, Hazuda HP, Patterson JK. Incidence of type II diabetes in Mexican-Americans predicted by fasting insulin and glucose levels, obesity and body-fat distribution. Diabetes 1990;39:283-8.

25. Haffner SM, D'Agostino R, Saad MF, Rewers M, Mykkänen L, Selby J, et al. Increased insulin resistance and insulin secretion in non-diabetic African-Americans and Hispanics compared with Non-Hispanics whites: the Insulin Resistance Atherosclerosis Study. Diabetes 1996:45:742-8.

26. Osei K, Schuster DP, Owusu SK, Amoah AGB. Race and ethnicity determine serum insulin and C-peptide concentration and hepatic insulin extraction and insulin clearance: comparative studies of three populations of West African ancestry and white Americans. Metab Clin Exp 1997:46:53-8.

27. Svec F, Nastasi K, Hilton C, Bao W, Srinivasan SR, Berenson GS. Black-white contrasts in insulin levels during pubertal development: the Bogalusa Heart Study. Diabetes 1992:41:313-7.

28. Pinhas-Hamiel O, Zeitler $P$. Insulin resistance, obesity and related disorders among black adolescents. J Pediatr 1996: 129:319-21.

29. Arslanian S. Insulin secretion and sensitivity in health African-American versus American white children. Clin Pediatr 1998:37:81-8.

30. Expert Committee on the diagnosis and classification of diabetes mellitus. Report of the Expert Committee on the diagnosis and classification of diabetes mellitus. Diabetes Care 1997:20:1 183-97.

31. Mannucci E, Bardini $G$, Ognibene A, Rotella CM. Comparison of ADA and WHO screening methods for diabetes mellitus in obese patients. Diabet Med 1999;16: $579-85$.

\section{Endereço para correspondência:}

Leila Maria Batista Araújo

Av. Sete de Setembro, 2417 ap. 601

40080-003 Salvador, BA

Fax: (071) 247-8492

e.mail: Imba@ufba.br 